\title{
Comparison of Efficacy of Systemic Therapies in Advanced Hepatocellular Carcinoma: Updated Systematic Review and Frequentist Network Meta-Analysis of Randomized Controlled Trials
}

This article was published in the following Dove Press journal:

Journal of Hepatocellular Carcinoma

\author{
Robin Park' \\ Laercio Lopes da Silva' \\ Voravech Nissaisorakarn' \\ Ivy Riano' \\ Stephen Williamson ${ }^{2}$ \\ Weijing Sun ${ }^{2}$ \\ Anwaar Saeed $\mathbb{I D}^{2}$ \\ 'Department of Medicine, MetroWest \\ Medical Center/Tufts University School of \\ Medicine, Framingham, MA, USA; \\ ${ }^{2}$ Department of Medicine, Division of \\ Medical Oncology, Kansas University \\ Cancer Center, Kansas City, KS, USA
}

Correspondence: Anwaar Saeed

Kansas University Cancer Center, GI

Oncology Program, Kansas City, KS, USA

Email asaeed@kumc.edu
Background: Several systemic agents have been approved for use in advanced hepatocellular carcinoma (aHCC). However, it is unclear which treatment is superior in either the firstor second-line settings due to the paucity of head-to-head comparative trials. Therefore, we have conducted a systematic review and network meta-analysis for the indirect comparison of the systemic agents in the first line and second line settings.

Methods: Randomized clinical trials evaluating systemic agents in first and second line settings in aHCC from inception to April 2020 were identified by searching PubMed, EMBASE, and Cochrane Databases and the annual ASCO and ESMO conferences from 2017 to 2020. Studies in English reporting clinical outcomes including overall survival (OS), progression-free survival (PFS), and objective response rate (ORR) were included. The primary outcomes of interest were pooled hazard ratios (HR) of OS and pooled odds ratios (OR) of ORR in first line studies and pooled HR of PFS and OR of ORR for second line studies. Additionally, OS for second line agents were reported in the qualitative analysis.

Results: Overall, first line studies comprised 8335 patients (13 studies) and second line studies comprised 4612 patients (11 studies). In the first line setting, atezolizumab plus bevacizumab was associated with the highest OS benefit over sorafenib (HR 0.58, 95\% CI, 0.42-0.80; P-score 0.993). Additionally, lenvatinib was associated with the greatest ORR benefit (OR 3.34, 95\% CI, 2.17-5.14; P-score 0.080) in the first line setting. In the second line setting, cabozantinib was associated with the highest PFS benefit over placebo (HR 0.44 , 95\% CI, 0.29-0.66; P-score 0.854) as well as the highest ORR benefit (OR 9.40, 95\% CI, 1.25-70.83, P-score, 0.266).

Conclusion: Atezolizumab plus bevacizumab appears to have superior efficacy among first line agents whereas cabozantinib appears to be superior in the second line setting. Further studies are warranted to determine whether the type of prior therapy received affects the efficacy of subsequent second line therapy.

Keywords: advanced hepatocellular carcinoma, targeted therapy, immunotherapy, systemic therapy

\section{Introduction}

Hepatocellular carcinoma (HCC) is the fourth most common cause of cancer-related mortality worldwide and accounts for $>80 \%$ of primary liver cancers. ${ }^{1,2}$ Treatment options for patients with very early, early, or intermediate stage (0-B) Barcelona Clinic 
Liver Cancer (BCLC) stage HCC include curative resection, liver transplantation, radiofrequency ablation, and chemoembolization. For patients with advanced (C) BCLC stage HCC (aHCC), defined by either portal invasion or extrahepatic disease spread and preserved liver function with an Eastern Cooperative Oncology Group Performance Score (ECOG PS) 1-2, systemic therapy remains the only option. ${ }^{3,4}$ Such patients with aHCC suffer from a poor prognosis with an estimated median overall survival (mOS) of 15 months. ${ }^{5}$

Systemic therapeutic agents currently approved for use or are currently being tested in aHCC can be largely divided into three classes. First, there are the tyrosine kinase inhibitors (TKI) with broad affinity to various receptor tyrosine kinases including the vascular endothelial growth factor receptor (VEGFR). The agents in this multi-target TKI class of drugs include sorafenib, lenvatinib, cabozantinib, and regorafenib. Second, there are targeted agents that inhibit the VEGF/VEGFR pathway which may be either TKIs that target the kinase domain of VEGFR or monoclonal antibodies against either VEGF or VEGFR. These include bevacizumab (anti-VEGF-A), apatinib (VEGFR2 TKI), and ramucirumab (anti-VEGFR2). Third, there are the immune checkpoint inhibitors against either the B7/CTLA-4 or PD-1/ L1 axis which aim to reinvigorate anti-tumor immune responses. These include the anti-PD-1 antibodies, nivolumab, pembrolizumab, and camrelizumab, the anti-PD-L1 antibodies, durvalumab, atezolizumab, and avelumab, and the anti-CTLA-4 antibodies, ipilimumab and tremelimumab.

Therapy options for aHCC had remained limited until sorafenib's approval in 2008, based on the SHARP trial. ${ }^{6}$ Since then, in the first line setting, lenvatinib was approved after the demonstration of non-inferiority to sorafenib in 2018, and atezolizumab plus bevacizumab was approved after demonstration of superiority to sorafenib in 2020. However, a head-to-head trial of lenvatinib to atezolizumab plus bevacizumab has not been conducted. Furthermore, several treatment options are currently approved for use in the second line setting, including nivolumab, nivolumab plus ipilimumab, pembrolizumab, cabozantinib, regorafenib, and ramucirumab based on placebo-controlled trials. ${ }^{7}$ No head-to-head trials have been conducted comparing second line treatments with each other, which makes the initial second line treatment choice difficult. Therefore, herein we have conducted a network meta-analysis of first- and second line systemic treatments in aHCC for indirect comparison of treatments that have been evaluated in randomized clinical trials.

\section{Methods}

\section{Protocol and Registration}

The following systematic review and network-meta-analysis are registered in PROSPERO (CRD42020179700).

\section{Database Search}

The search was conducted in PubMed, EMBASE, and the Cochrane Database published from conception to search date (June 8th, 2020). Also, abstracts and posters from the ASCO (Annual Meeting, SITC Clinical Immuno-oncology Symposium, and Gastrointestinal Cancers Symposium) and ESMO (main annual) conferences from the last 3 years from the search date (ie, 2018, 2019, 2020) were included. The initial search was supplemented with a follow-up search for studies newly published during this interval period. Attempts were made to contact authors via e-mail whenever additional data from included studies needed to be obtained. The following is an example of the search strategy for a selected database (ie, PubMed). The search was conducted using the keywords "hepatocellular carcinoma" AND ("systemic therapy" OR "clinical trials" OR "targeted agents" OR "immune therapy"). The search was limited to publications fulfilling the following criteria - studies conducted in human subjects, ages 19+, and published in English. Search results were combined into Endnote Online then duplicate studies were removed using the "remove duplicates" function.

\section{Study Selection}

The criteria for inclusion and exclusion of studies were established before study selection. The following inclusion criteria were applied: (1) randomized-controlled studies with head-to-head comparisons of at least two treatment arms; (2) systemic therapy in the first line or second line or later setting for advanced or metastatic HCC; and (3) at least one of the following clinical outcomes reported odds ratios (OR) and $95 \%$ confidence intervals (95\% CI) for objective response rate (ORR) based on response evaluation criteria in solid tumors (RECIST), modified RECIST (mRECIST), or immune-related RECIST (iRECIST/irRECIST); and hazard ratios (HR) and 95\% $\mathrm{CI}$ for OS and PFS. If only raw data were available for ORR, OR and $95 \%$ CI were calculated using a univariate model and included in the analysis ( 0.5 was added to zero cells for computation). Furthermore, where PFS was not reported, time to progression (TTP) was used in place $(\mathrm{n}=1$, EVOLVE-1). The following exclusion criteria were applied: review articles, systematic reviews and meta- 
analyses, case reports, case series, and retrospective analyses. The initial screening based on the title and abstract was followed by a full review of the manuscripts and was conducted in tandem by RP and LL. Any discrepancy in the list of selected studies was resolved with mediation by VB. When data overlapped, the study with the most recent or comprehensive data was selected for inclusion.

\section{Data Extraction and Quality Assessment}

The authors created a data extraction form with pre-specified items for study characteristics and clinical outcomes. Items related to study characteristics included the following: (1) first author; (2) year of publication; (3) trial name or national clinical trial (NCT) identification number; (4) trial phase; (5) version of RECIST, mRECIST, iRECIST, or irRECIST and CTCAE used; and (6) majority ethnicity of trial subjects; (7) country. Items related to clinical outcomes included the following: median OS, HR $(95 \% \mathrm{CI})$ for OS and PFS, and ORR.
The risk of bias for individual studies was assessed at the study level based on the Revised Cochrane risk-of-bias tool for randomized trials (RoB 2.0, version 22 August 2019).

\section{Statistical Analysis}

The network meta-analysis was conducted under the frequentist model using the software " $\mathrm{R}$ " with the package "netmeta". The meta-analysis was conducted using the random-effects model under the assumption of significant heterogeneity. Based on the I2 measure, heterogeneity was defined as low $(<25 \%)$, moderate $(25-50 \%)$, or high $(>75 \%)$. Network inconsistency was evaluated using the net-splitting method, and Net Rank Plot was used to provide an additional measure of comparison. For HR of OS and PFS, a higher P-score represented a greater probability of prolonged OS and PFS, whereas a lower P-score represented a greater probability of objective response. The risk of bias across studies was assessed graphically and

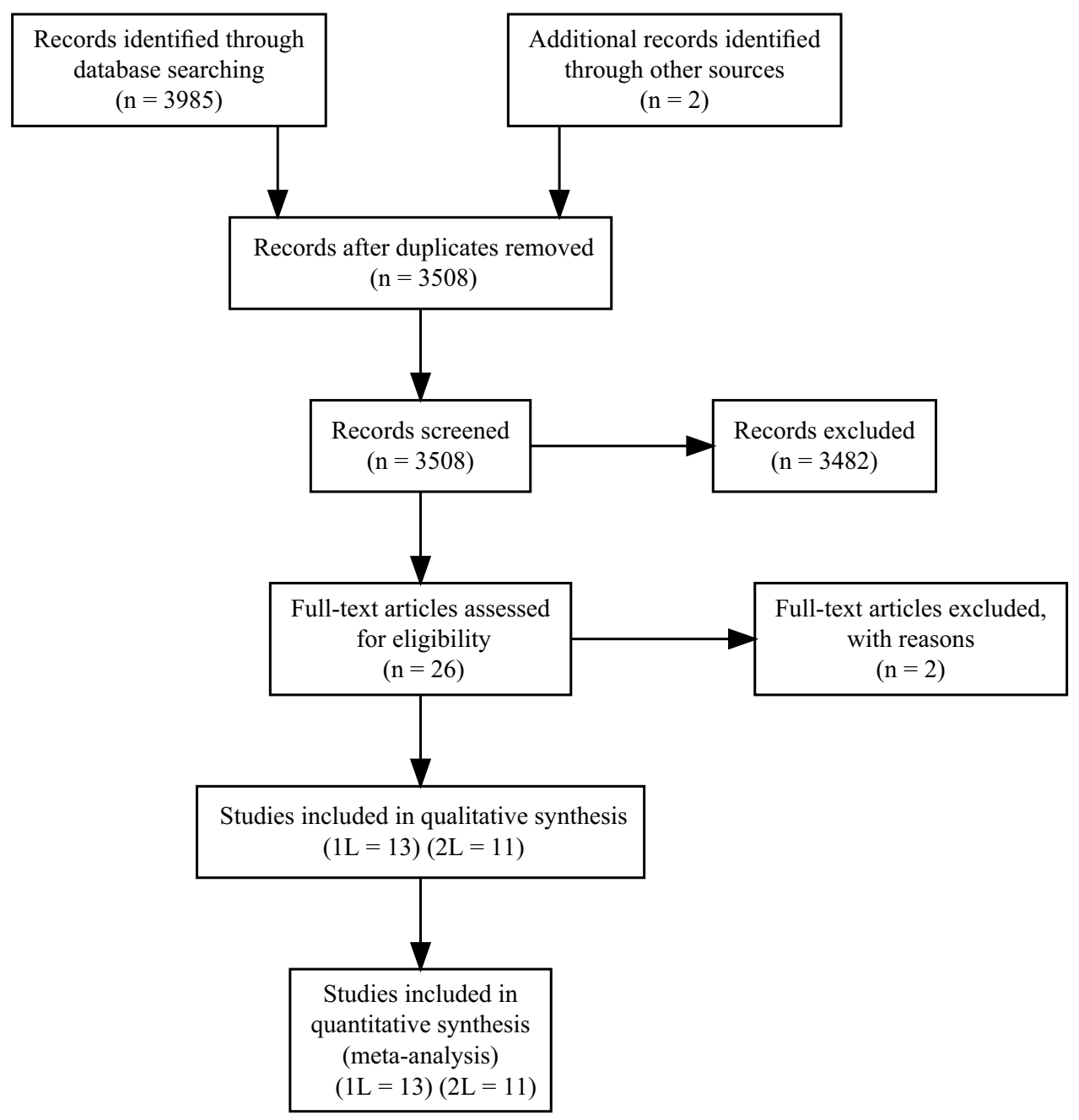

Figure I PRISMA flow diagram for database search. 
numerically using the funnel plot and Egger's regression test. A quantitative meta-analysis was conducted for HR of OS and OR of ORR for first line trials. For second line trials, HR of PFS and OR of ORR were subjects of quantitative analysis. Of note, a quantitative meta-analysis was not conducted for HR of OS for second line trials given the presence of non-mature follow-up data in studies that may introduce bias and result in erroneous conclusions and a qualitative meta-analysis was conducted instead.

\section{Results}

\section{Characteristics of Included Studies}

In total, 3508 studies were screened, 26 full-text articles were assessed for eligibility, and 13 first line and 11 second line trials were included in the final quantitative and qualitative synthesis (Figure 1). The treatment network consisted of 13 double-arm studies for the first line trials, with most studies featuring sorafenib as the comparative arm (Figure 2A). For second line trials, the treatment network consisted of 11 placebo-controlled studies (Figure 2B). Median follow-up ranged 8.9 to 27.7 months and 5.5 to 24.6 months for the first line and second line trials, respectively. Almost all studies were conducted in multinational settings (first line, $\mathrm{n}=12$ studies; second line, $\mathrm{n}=10$ studies) and were Phase III trials (first line, $\mathrm{n}=11$ studies; second line, $\mathrm{n}=9$ studies). Six trials featured a majority Caucasian population, while the rest of the trials consisted of majority Asian populations. Of note, two trials were conducted in a biomarker-selected patient population (REACH-II, AFP; NCT01755767, MET) (Supplemental Tables 1 and 2).

\section{Qualitative Systematic Review}

Among the identified first line randomized trials, four trials had tested agents that are currently approved for use or recommended by major guidelines in aHCC. First, the SHARP trial was the first study to demonstrate the efficacy of a systemic agent over placebo. This trial randomized 602 treatment-naïve aHCC patients to either placebo or sorafenib. Data showed that mOS was significantly improved over placebo. Second, the REFLECT trial compared lenvatinib against sorafenib as the standard of care. This study met its primary endpoint of demonstrating the non-inferiority of Lenvatinib to sorafenib and led to the approval of lenvatinib for first line therapy. Third, the CheckMate- 459 trial compared nivolumab to sorafenib in 743 treatment-naïve patients. Although nivolumab did not meet statistical significance for its primary endpoint of OS, it did demonstrate tendency towards improved OS and ORR. Fourth, the most recent IMBrave150 trial compared atezolizumab plus bevacizumab to sorafenib in 485 patients. This study, for the first time, demonstrated the superiority of a novel systemic therapy regimen over sorafenib in prolonging OS.

Of the second line randomized trials, four trials have led to the approval of systemic agents for use in aHCC. First, the RESORCE trial compared regorafenib to placebo among 573 previously treated patients and demonstrated the superior OS and PFS of regorafenib. Second, the CELESTIAL trial compared cabozantinib to placebo among 707 previously treated

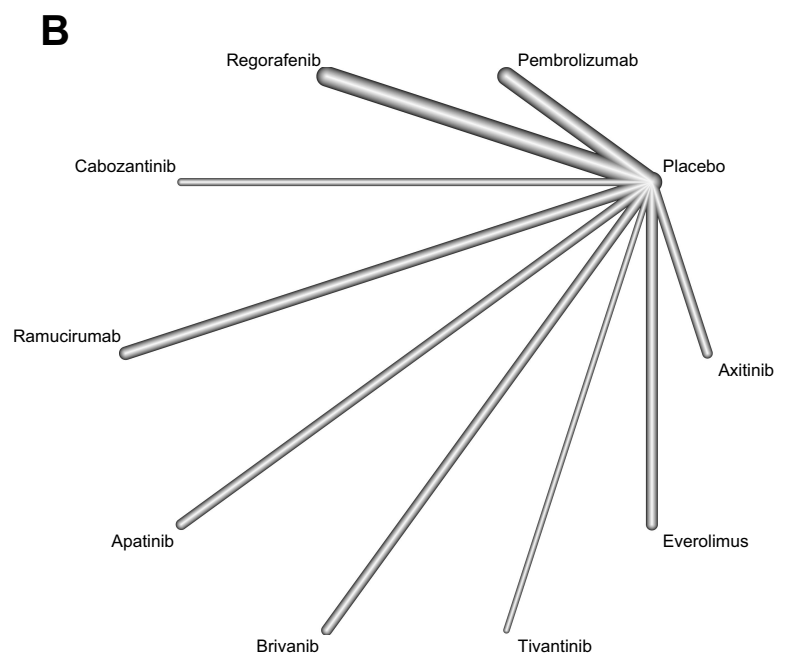

Figure 2 Net graph diagram. (A) First line trials. (B) Second line trials. 
patients and also demonstrated the superior OS and PFS of this agent. Third, the KEYNOTE-240 trial demonstrated the superior OS and PFS of pembrolizumab to placebo in previously treated patients. Fourth, the REACH and REACH-2 trials led to the approval of ramucirumab. Whilst ramucirumab failed to show superiority to placebo in the REACH trial, an exploratory subgroup analysis showed that in patients with high AFP (AFP > 400), ramucirumab showed superior efficacy over placebo. This led to the REACH-II trial which subsequently showed that ramucirumab prolonged PFS and OS over placebo in previously treated aHCC patients.

A

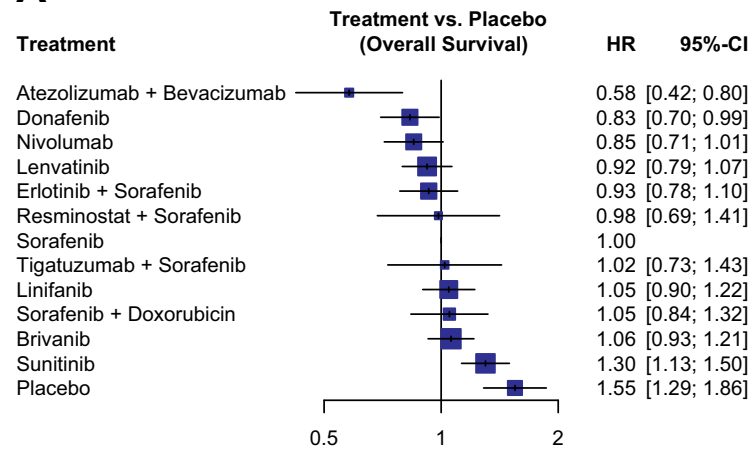

\section{Network Meta-Analysis of First Line Trials}

Atezolizumab plus bevacizumab was associated with the greatest OS benefit (HR, $0.58,95 \%$ CI $0.42-0.80$ ) and the highest P-score (0.993) (Figure 3A, Supplemental Figure 4A). No agents other than atezolizumab plus bevacizumab and donafenib were superior to sorafenib. Also, atezolizumab plus bevacizumab was superior to all other agents including lenvatinib and nivolumab except donafenib (Figure 3C).

Lenvatinib was associated with the greatest ORR benefit (OR, 3.34, 95\% CI 2.17-5.14) with the highest P-score

B

Treatment
Lenvatinib
Atezolizumab + Bevacizumab
Nivolumab
Linifanib
Sorafenib + Doxorubicin
Erlotinib + Sorafenib
Donafenib
Brivanib
Sunitinib
Sorafenib
Placebo

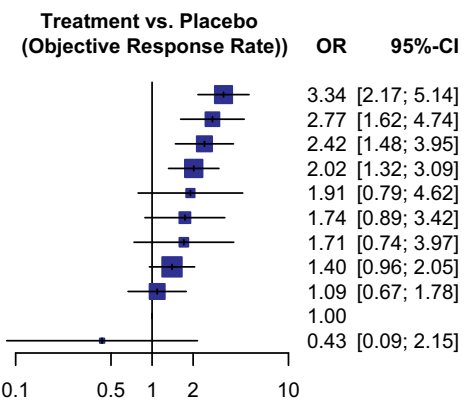

C

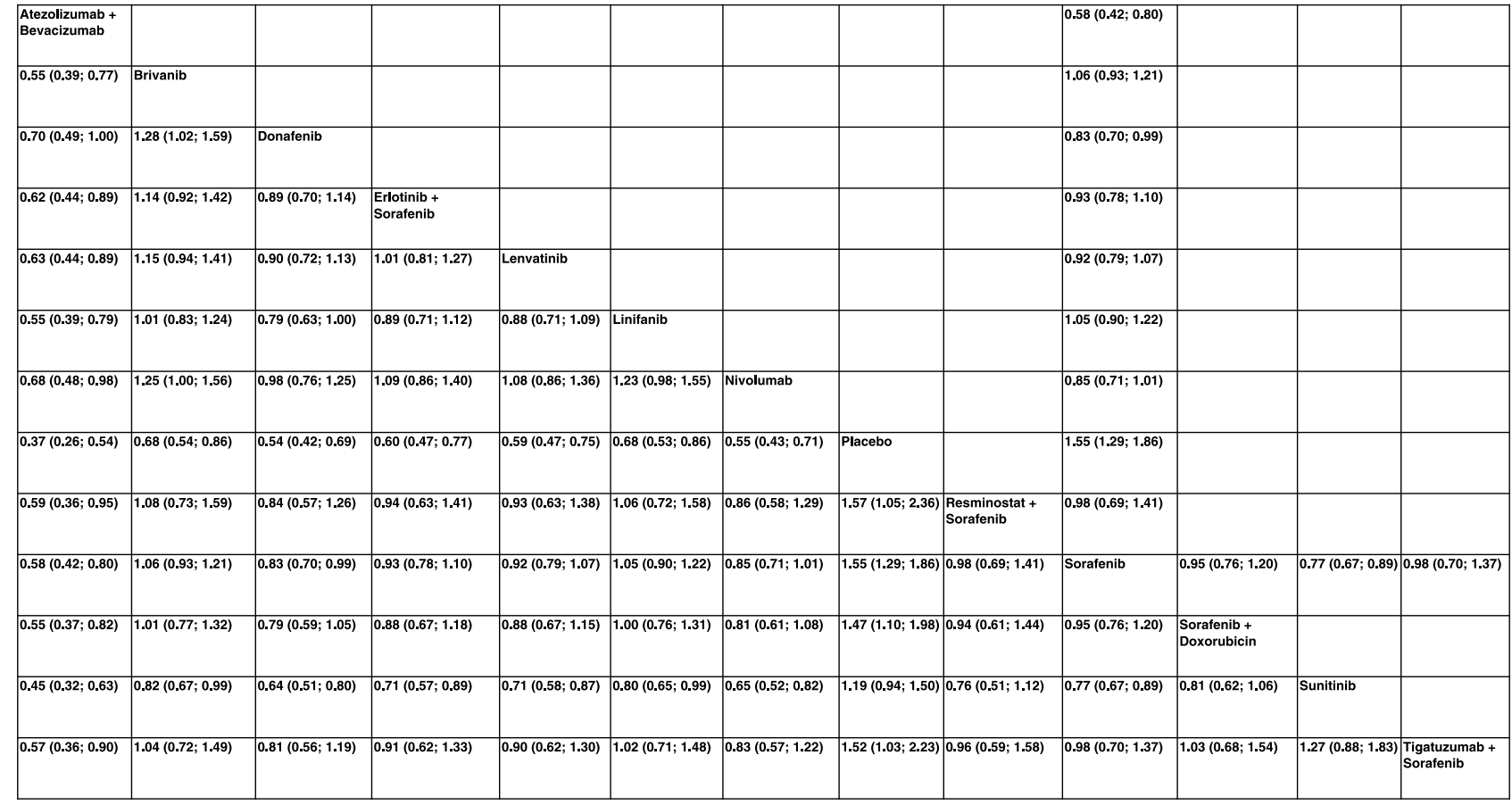

Figure 3 Continued. 


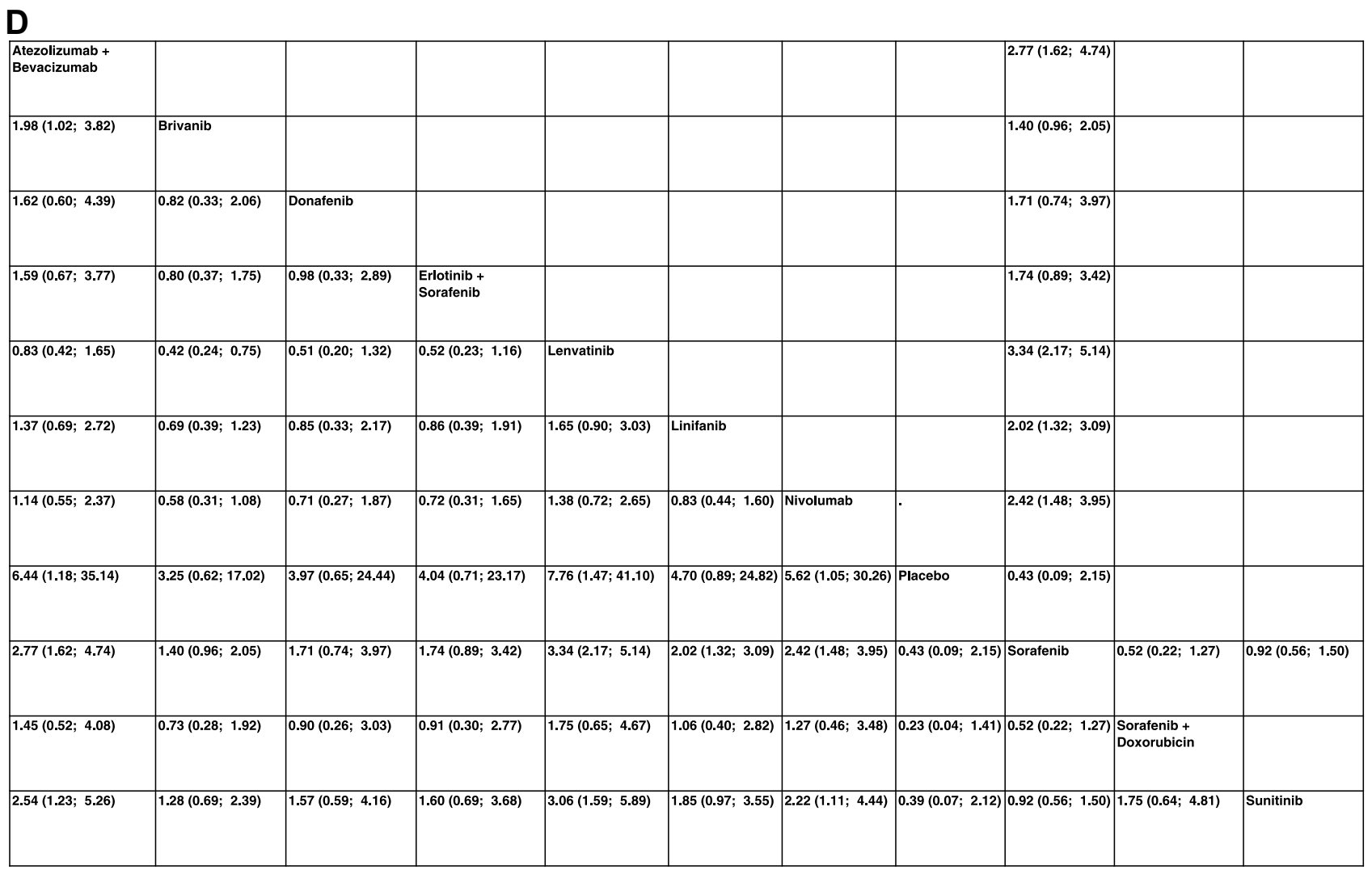

Figure 3 Network meta-analysis of first line trials. (A) Forest plot for HR of OS. (B) Forest plot for OR of ORR. (C) Net league graph for indirect comparisons for HR of OS. (D) Net league graph for indirect comparisons for OR of ORR.

(0.080) (Figure 3B, Supplemental Figure 4B). Atezolizumab plus bevacizumab, lenvatinib, and nivolumab were all associated with greater ORR compared to sorafenib. Of note, donafenib was non-superior to sorafenib in terms of ORR (OR, 1.71, 95\% CI 0.74-3.97). No significant differences for ORR were seen among atezolizumab plus bevacizumab, donafenib, lenvatinib, and nivolumab (Figure 3D).

\section{Network Meta-Analysis of Second Line Trials}

Cabozantinib demonstrated the greatest PFS benefit (HR, $0.44,95 \%$ CI $0.29-0.66)$ and the highest P-score (0.854) (Figure 4A, Supplemental Figure 5A). Also, all nine of the studied agents showed a PFS benefit over placebo. Of note, cabozantinib and apatinib showed a favorable PFS benefit over pembrolizumab in indirect comparison (cabo vs pemb, HR $0.56,95 \%$ CI $0.36-0.89$; apat vs pemb, HR $0.60,95 \%$ CI $0.39-0.93$ ) (Figure 4C).

Cabozantinib was also associated with the greatest ORR benefit in terms of $\mathrm{HR}(9.40,95 \%$ CI $1.25-70.83)$ (Figure 4B, Supplementary Figure 5B). Also, six agents demonstrated an ORR benefit over placebo (cabozantinib, pembrolizumab, ramucirumab, apatinib, brivanib, and regorafenib) (Figure 4D). No significant benefit was demonstrated by cabozantinib over any of the other agents in indirect comparison. Net splitting plots were limited by the paucity of studies evaluating direct comparisons between agents as all included studies were placebo-controlled trials. Third, five systemic agents demonstrated an OS benefit over placebo (regorafenib, ramucirumab, cabozantinib, apatinib, and pembrolizumab) (Supplemental Table 2).

\section{Assessment of Bias}

Included studies were found to have a low risk of bias across all 5 domains (Supplemental Figure 1A and B). The funnel plot and Egger's test revealed no significant bias across studies in the first line $(p=0.505)$ and the second line settings $(p=0.574)$ (Supplemental Figures 2 and 3$)$.

\section{Discussion}

Herein we demonstrate that atezolizumab plus bevacizumab is associated with the most significant OS benefit in 
the first line setting and that cabozantinib is associated with the most significant PFS benefit in the second line setting. Also, lenvatinib was associated with the greatest ORR benefit in the first line setting.

It should be noted that while atezolizumab plus bevacizumab had the highest-ranking per P-score for OS benefit in the first line setting, an overlap of $95 \%$ confidence interval was present with several other agents, including donafenib, nivolumab, and lenvatinib. Nonetheless, the greater magnitude of HR for OS does suggest atezolizumab plus bevacizumab may be superior to the other treatments. The success of this anti-VEGF and ICI combination is in keeping with the move towards combination therapy evaluations in clinical trials where this specific combination is currently the most frequently evaluated regimen. ${ }^{8}$ For instance, the COSMIC021 trials have demonstrated promising efficacy data for atezolizumab and cabozantinib in prostate cancer, nonsmall cell lung cancer, renal cell carcinoma, and urothelial carcinoma, ${ }^{9-12}$ and the CAMILLA study has demonstrated the potential efficacy of durvalumab with cabozantinib in advanced GI cancers. ${ }^{13}$ Furthermore, a recent, multi-center, Phase II trial tested the safety and efficacy of a camrelizumab (anti-PD-1 antibody) plus apatinib (anti-VEGFR-2) in aHCC patients who were either treatment-naïve or refractory to first
A

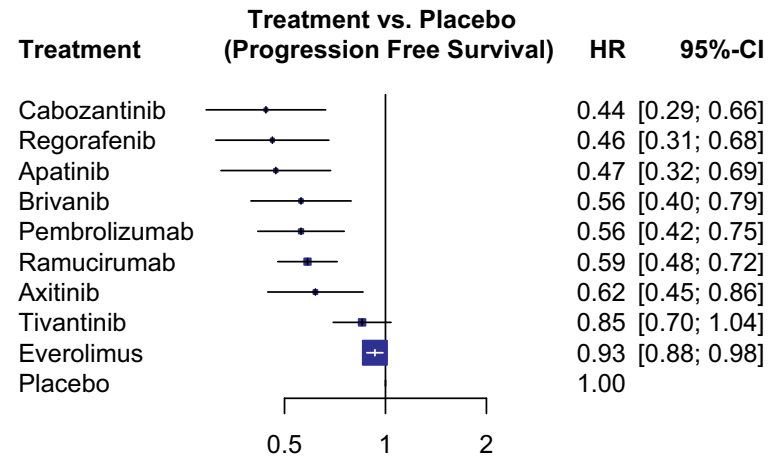

B

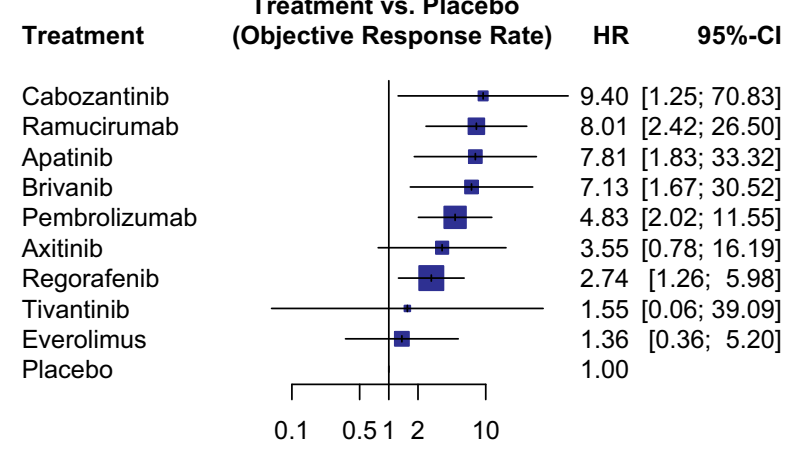

\section{c}

\begin{tabular}{|c|c|c|c|c|c|c|c|c|c|}
\hline Apatinib & & & & & & $0.47(0.32 ; 0.69)$ & & & \\
\hline $0.76(0.46 ; 1.25)$ & Axitinib & & & & & $0.62(0.45 ; 0.86)$ & & & \\
\hline $0.84(0.51 ; 1.40)$ & $1.10(0.69 ; 1.77)$ & Brivanib & & & & $0.56(0.40 ; 0.79)$ & & & \\
\hline $1.07(0.61 ; 1.87)$ & $1.40(0.83 ; 2.37)$ & $1.27(0.75 ; 2.17)$ & Cabozantinib & & & $0.44(0.29 ; 0.66)$ & & & \\
\hline $0.51(0.35 ; 0.74)$ & $0.66(0.48 ; 0.93)$ & $0.60(0.42 ; 0.85)$ & $0.47(0.31 ; 0.72)$ & Everolimus & & $0.93(0.88 ; 0.98)$ & & & \\
\hline $0.84(0.52 ; 1.36)$ & $1.10(0.71 ; 1.72)$ & $1.00(0.63 ; 1.57)$ & $0.79(0.47 ; 1.30)$ & $1.66(1.23 ; 2.25)$ & Pembrolizumab & $0.56(0.42 ; 0.75)$ & & & \\
\hline $0.80(0.52 ; 1.23)$ & $1.06(0.72 ; 1.55)$ & $0.96(0.64 ; 1.43)$ & $0.75(0.48 ; 1.19)$ & $1.59(1.29 ; 1.96)$ & $0.96(0.67 ; 1.37)$ & $1.71(1.39 ; 2.09)$ & Ramucirumab & & \\
\hline $1.02(0.60 ; 1.76)$ & $1.34(0.81 ; 2.23)$ & $1.22(0.72 ; 2.04)$ & $0.96(0.54 ; 1.68)$ & $2.02(1.37 ; 2.99)$ & $1.22(0.75 ; 1.98)$ & $2.17(1.47 ; 3.20)$ & $1.27(0.82 ; 1.97)$ & Regorafenib & \\
\hline $0.55(0.36 ; 0.85)$ & $0.73(0.49 ; 1.06)$ & $0.66(0.44 ; 0.98)$ & $0.52(0.33 ; 0.82)$ & $1.09(0.89 ; 1.34)$ & $0.66(0.46 ; 0.94)$ & $1.17(0.96 ; 1.43)$ & $0.69(0.52 ; 0.91)$ & $0.54(0.35 ; 0.84)$ & Tivantinib \\
\hline
\end{tabular}

Figure 4 Continued. 
D

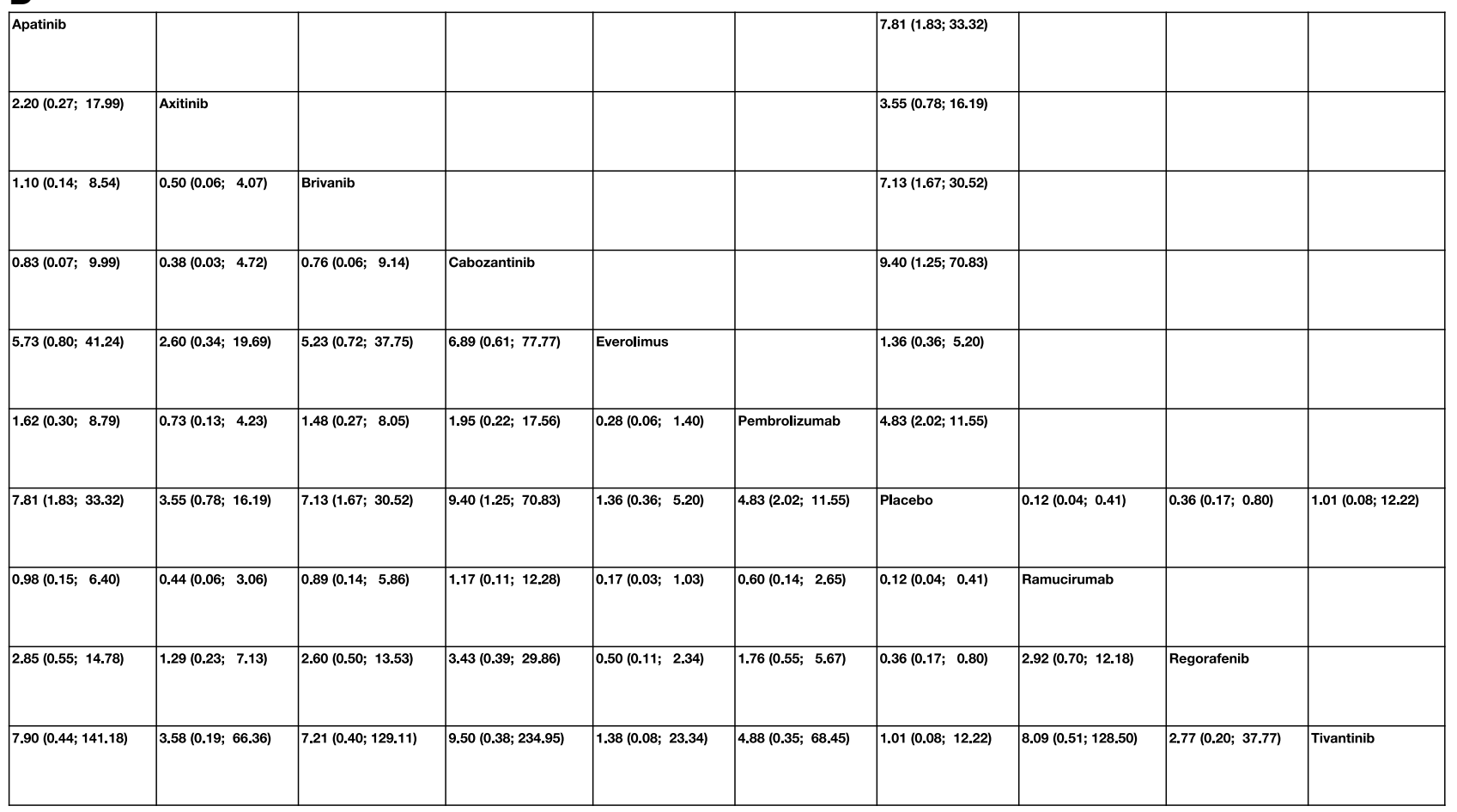

Figure 4 Network meta-analysis of second line trials. (A) Forest plot for HR of OS. (B) Forest plot for OR of ORR. (C) Net league graph for indirect comparisons for HR of OS. (D) Net league graph for indirect comparisons for OR of ORR.

line therapy. The data showed promising efficacy including an ORR of $34.3 \%$ in the first line and $22.5 \%$ in the second line settings; furthermore, the mPFS were 5.7 and 5.5 months in the first- and second line settings, respectively. The toxicity was high as expected with an ICI plus anti-VEGF combination, with grade 3 or higher adverse events occurring in $77.4 \%$ of patients. ${ }^{14}$ Thus, targeted agents against the VEGF/VEGFR pathway are powerful partners to ICIs in combination regimens and more such combination therapies will likely demonstrate efficacy in aHCC.

The results of two second line studies were notably excluded in this analysis due to their absence of comparative arms. In the CheckMate-040 trial, which evaluated nivolumab with or without ipilimumab, nivolumab monotherapy demonstrated an ORR of $14 \%$ and an mOS of 15.1 months in aHCC patients who progressed or were intolerant of sorafenib. ${ }^{15}$ Also, nivolumab plus ipilimumab demonstrated an ORR of $31-32 \%$, an mDOR of 16.622.2 months, and an mOS of 12.5-22.8 months. ${ }^{16}$ In a recent trial presented at GI-ASCO 2020 (NCT02519348), tremelimumab plus durvalumab demonstrated an ORR of 9.5-22.7\%, mDOR of 13.2 months to not reached, and mOS of 11.3-18.7 months after 11.7 months of median follow up. ${ }^{17}$ In comparison, nivolumab monotherapy appears comparable while nivolumab with ipilimumab and tremelimumab with durvalumab appear superior to pembrolizumab monotherapy, although the indirect comparison was not conducted.

Another interesting question that arises in the sequencing of second line therapies is whether the sequence of TKI to ICI or ICI to TKI makes a difference in the treatment effect. A recent, non-randomized, phase II trial testing avelumab (anti-PD-L1 antibody) in aHCC patients who were previously treated with sorafenib suggested that there may be an association between prior sorafenib response and subsequent ICI response. The data showed that patients who received a longer duration of sorafenib compared to those who received a shorter duration had a higher disease control rate, PFS, and OS. ${ }^{18}$ Although this study was limited by the small number of patients $(\mathrm{N}=30)$, the results suggest further investigation into the effects of treatment sequencing and prior treatment response on the subsequent treatment effect.

Our findings suggest that atezolizumab plus bevacizumab should be the initial choice for first line therapy in aHCC in patients who are candidates for receiving immune 
checkpoint inhibitor therapy. On the other hand, patients who are ineligible for immune checkpoint inhibitor therapy due to underlying conditions, such as active autoimmune disorders, should be considered for either sorafenib or lenvatinib. Although donafenib appears to be another favorable option in this setting, the agent is currently not FDA approved. In the second line setting, cabozantinib, regorafenib, pembrolizumab appear to be favorable options. Also, while apatinib appears comparable to these options, it is currently an FDA approved agent.

This study has several limitations. First, given that direct evidence holds greater weight than indirect evidence, the paucity of direct evidence among both the first line and second line trials due to most comparison arms featuring sorafenib in the former and placebo in the latter limited the power of this analysis. Second, a quantitative meta-analysis for OS for second line studies was not conducted, and a follow-up study is warranted to evaluate this outcome. Third, the dependence of efficacy of second line treatments on the type of the first line agent received is a topic of great interest, especially with the increasing use of ICI-based treatments in the first line setting. However, due to the limited published data in this regard, a subgroup analysis was not conducted. Therefore, future studies are warranted to address this question.

\section{Conclusions}

The combination atezolizumab plus bevacizumab appears to be the most favorable choice for first line treatment. In the second line setting, cabozantinib, regorafenib, and pembrolizumab appear to be comparably favorable treatment options.

\section{Acknowledgments}

The abstract of this paper was presented at the ASCO GI 2021 as a poster presentation with interim findings. The poster's abstract was published in 'Poster Abstracts' in ASCO: https://ascopubs.org/doi/abs/10.1200/JCO.2021. 39.3_suppl.293.

\section{Funding}

The authors did not receive any specific grant from funding agencies in the public, commercial, or not-for-profit sectors for this research.

\section{Disclosure}

Anwaar Saeed reports research funding (to institution) from AstraZeneca, Exelixis, Bristol Myers Squibb, Clovis, and Merck; advisory board/consulting fees from
Bristol Myers Squibb, AstraZeneca, Pfizer, and Exelixis. Stephen Williamson reports research funds from Daiichi Sankyo for Bayer Health, Acceleron Pharma, Sotio, Rogosin, Merck Serono EMD Serono, Sanofi, Novartis, Nektar, Seattle Genetics, Astellas Pharma, Aleon, BristolMyers Squibb, Regeneron; for more open payments data, it can be obtained from https:/openpaymentsdata.cms.gov/ physician/1092063/summary. The remaining authors report no conflicts of interest in this work.

\section{References}

1. El-Serag HB, Rudolph KL. Hepatocellular carcinoma: epidemiology and molecular carcinogenesis. Gastroenterology. 2007;132:25572576. doi:10.1053/j.gastro.2007.2504.2061

2. Fitzmaurice C, Allen C, Barber RM, et al. Global, regional, and national cancer incidence, mortality, years of life lost, years lived with disability, and disability-adjusted life-years for 32 cancer groups, 1990 to 2015: a systematic analysis for the Global Burden of Disease Study. JAMA Oncol. 2017;3:524-548. doi:10.1001/jamaoncol.2016.5688

3. Llovet JM, Di Bisceglie AM, Bruix J, et al. Design and endpoints of clinical trials in hepatocellular carcinoma. J Natl Cancer Inst. 2008;100:698-711. doi:10.1093/jnci/djn1134

4. Yang JD, Hainaut P, Gores GJ, Amadou A, Plymoth A, Roberts LR. A global view of hepatocellular carcinoma: trends, risk, prevention and management. Nat Rev Gastroenterol Hepatol. 2019;16:589-604. doi:10.1038/s41575-41019-40186-y

5. Park JW, Chen M, Colombo M, et al. Global patterns of hepatocellular carcinoma management from diagnosis to death: the BRIDGE Study. Liver Int. 2015;35:2155-2166. doi:10.1111/liv.12818

6. Lang L. FDA approves sorafenib for patients with inoperable liver cancer. Gastroenterology. 2008;134:379. doi:10.1053/j.gastro.2007.12.037

7. Gordan JD, Kennedy EB, Abou-Alfa GK, et al. Systemic therapy for advanced hepatocellular carcinoma: ASCO guideline. J Clin Oncol. 2020;16:02672.

8. Upadhaya S, Neftelino ST, Hodge JP, Oliva C, Campbell JR, Yu JX. Combinations take centre stage in PD1/PDL1 inhibitor clinical trials. Nat Rev Drug Discov. 2020;11:020-00204.

9. Agarwal N, Loriot Y, McGregor BA, et al. Cabozantinib in combination with atezolizumab in patients with metastatic castration-resistant prostate cancer: results of cohort 6 of the COSMIC-021 study. J Clin Oncol. 2020;38:5564. doi:10.1200/JCO.2020.38.15_supp1.5564

10. Choueiri TK, Albiges L, Powles T, Scheffold C, Wang F, Motzer RJ. A phase III study (COSMIC-313) of cabozantinib (C) in combination with nivolumab (N) and ipilimumab (I) in patients (pts) with previously untreated advanced renal cell carcinoma (aRCC) of intermediate or poor risk. J Clin Oncol. 2020;38:TPS767-TPS767. doi:10.1200/JCO.2020.38.6_suppl.TPS767

11. Neal JW, Lim FL, Felip E, et al. Cabozantinib in combination with atezolizumab in non-small cell lung cancer (NSCLC) patients previously treated with an immune checkpoint inhibitor: results from cohort 7 of the COSMIC-021 study. J Clin Oncol. 2020;38:9610. doi:10.1200/JCO.2020.38.15_suppl.9610

12. Pal SK, Agarwal N, Loriot $\mathrm{Y}$, et al. Cabozantinib in combination with atezolizumab in urothelial carcinoma previously treated with platinum-containing chemotherapy: results from cohort 2 of the COSMIC-021 study. $J$ Clin Oncol. 2020;38:5013. doi:10.1200/JCO.2020.38.15_suppl.5013

13. Saeed A, Phadnis M, Park R, et al. Cabozantinib (cabo) combined with durvalumab (durva) in gastroesophageal (GE) cancer and other gastrointestinal (GI) malignancies: preliminary phase Ib CAMILLA study results. J Clin Oncol. 2020;38:4563. doi:10.1200/ JCO.2020.38.15_suppl.4563 
14. El-Khoueiry AB, Melero I, Yau TC, et al. Impact of antitumor activity on survival outcomes, and nonconventional benefit, with nivolumab (NIVO) in patients with advanced hepatocellular carcinoma (aHCC): subanalyses of CheckMate-040. J Clin Oncol. 2018;36:475. doi:10.1200/JCO.2018.36.4-suppl.475

15. Yau T, Kang YK, Kim TY, et al. Efficacy and safety of nivolumab plus ipilimumab in patients with advanced hepatocellular carcinoma previously treated with sorafenib: the check mate 040 randomized clinical trial. JAMA Oncol. 2020;1.

16. Kelley RK, Sangro B, Harris WP, et al. Efficacy, tolerability, and biologic activity of a novel regimen of tremelimumab (T) in combination with durvalumab (D) for patients (pts) with advanced hepatocellular carcinoma (aHCC). J Clin Oncol. 2020;38:4508. doi:10.1200/JCO.2020.38.15_suppl.4508
17. Yau T, Park JW, Finn RS, et al. CheckMate 459: a randomized, multicenter phase III study of nivolumab (NIVO) vs sorafenib (SOR) as first-line (1L) treatment in patients (pts) with advanced hepatocellular carcinoma (aHCC). Ann Oncol. 2019;30:v874-v875. doi:10.1093/ annonc/mdz394.029

18. Finn RS, Qin S, Ikeda M, et al. Atezolizumab plus bevacizumab in unresectable hepatocellular carcinoma. $N$ Engl J Med. 2020;382:1894-1905. doi:10.1056/NEJMoa1915745

\section{Publish your work in this journal}

The Journal of Hepatocellular Carcinoma is an international, peerreviewed, open access journal that offers a platform for the dissemination and study of clinical, translational and basic research findings in this rapidly developing field. Development in areas including, but not limited to, epidemiology, vaccination, hepatitis therapy, pathology and molecular tumor classification and prognostication are all considered for publication. The manuscript management system is completely online and includes a very quick and fair peer-review system, which is all easy to use. Visit http://www.dovepress.com/ testimonials.php to read real quotes from published authors. 